\title{
Ablation index guided pulmonary vein isolation can reduce early recurrences of atrial tachyarrhythmias
}

\author{
Koji Yasumoto $^{1}$, Yasuyuki Egami ${ }^{1}$, Kohei Ukita ${ }^{1}$, Akito Kawamura ${ }^{1}$, Hitoshi Nakamura ${ }^{1}$, \\ Yutaka Matsuhiro ${ }^{1}$, Masaki Tsuda ${ }^{1}$, Naotaka Okamoto ${ }^{1}$, Akihiro Tanaka ${ }^{1}$, Yasuharu \\ Matsunaga-Lee $^{1}$, Masamichi Yano ${ }^{1}$, Ryu Shutta ${ }^{1}$, Yasushi Sakata ${ }^{2}$, Masami Nishino ${ }^{1}$, and \\ Jun Tanouchi ${ }^{1}$ \\ ${ }^{1}$ Osaka Rosai Hospital \\ ${ }^{2}$ Osaka University Graduate School of Medicine Department of Cardiovascular Medicine
}

September 11, 2020

\begin{abstract}
Introduction: Early recurrence of atrial tachyarrhythmias (ERAT) cause various symptoms and predict worse outcomes after pulmonary vein isolation (PVI). This study aimed to clarify whether ablation index (AI) guided PVI, which is a novel technology of radiofrequency ablation, could reduce ERAT as compared to conventional contact force (CF) guided PVI. Methods: Consecutive AF patients who underwent initial PVI from September 2014 to August 2019 were enrolled. We divided the patients into two groups: patients who underwent AI-guided PVI (AI group) and those who underwent CF-guided PVI (CF group). Using propensity score matching (PSM), we adjusted for the patient backgrounds. We compared the incidence of ERAT and late reconnection rate of isolated PVs during second session between the two groups. ERAT was defined as any atrial tachyarrhythmias [?]30 seconds during a 90-day blanking period. Results: A total of 697 patients (paroxysmal 51\%) were enrolled. After the PSM, both groups included 229 patients. The incidence of ERAT was significantly lower in AI group than $\mathrm{CF}$ group $(21.5 \%$ vs. $36.1 \%, \mathrm{P}<0.001)$. The rate of $\mathrm{PV}$ reconnection was lower in AI group than CF group (45.8\% vs. $71.4 \%, \mathrm{P}=0.028)$. Multivariate analysis demonstrated that AI-guided PVI was independently correlated with the incidence of ERAT $(\mathrm{OR}=0.415,95 \% \mathrm{CI}=0.269-0.639, \mathrm{P}<0.001)$. Conclusion: AI-guided PVI can reduce ERAT as compared to conventional CF-guided PVI.
\end{abstract}

Ablation index guided pulmonary vein isolation can reduce early recurrences of atrial tachyarrhythmias

Koji Yasumoto, MD,${ }^{1}$ Yasuyuki Egami, MD,${ }^{1}$ Kohei Ukita, MD, ${ }^{1}$ Akito Kawamura, MD,${ }^{1}$ Hitoshi Nakamura, $\mathrm{MD},{ }^{1}$ Yutaka Matsuhiro, MD, ${ }^{1}$

Masaki Tsuda, MD, ${ }^{1}$ Naotaka Okamoto, MD, ${ }^{1}$ Akihiro Tanaka, MD ${ }^{1}$

Yasuharu Matsunaga-Lee, MD, ${ }^{1}$ Masamichi Yano, MD, PhD,${ }^{1}$ Ryu Shutta, MD, ${ }^{1}$ Yasushi, Sakata, MD, PhD, FACC, FESC, ${ }^{2}$ Masami Nishino, MD, PhD, FACC, FESC, ${ }^{1}$ Jun Tanouchi, MD, PhD ${ }^{1}$

1: Department of Cardiology, Osaka Rosai Hospital, Sakai, Osaka, Japan 2: Department of Cardiovascular Medicine, Osaka University Graduate School of Medicine, Suita, Osaka, Japan

\section{Corresponding author:}

Masami Nishino, MD, PhD, FACC, FESC Division of Cardiology, Osaka Rosai Hospital, Sakai, Osaka, Japan 3-1179 Nagasonecho, kita-ku, Sakai, Osaka, Japan TEL: +81-72-252-3561 FAX: +81-72-255-3349 E-mail: mnishino@osakah.johas.go.jp 


\section{Structured Abstract}

Introduction: Early recurrence of atrial tachyarrhythmias (ERAT) cause various symptoms and predict worse outcomes after pulmonary vein isolation (PVI). This study aimed to clarify whether ablation index (AI) guided PVI, which is a novel technology of radiofrequency ablation, could reduce ERAT as compared to conventional contact force $(\mathrm{CF})$ guided PVI.

Methods: Consecutive AF patients who underwent initial PVI from September 2014 to August 2019 were enrolled. We divided the patients into two groups: patients who underwent AI-guided PVI (AI group) and those who underwent CF-guided PVI (CF group). Using propensity score matching (PSM), we adjusted for the patient backgrounds. We compared the incidence of ERAT and late reconnection rate of isolated PVs during second session between the two groups. ERAT was defined as any atrial tachyarrhythmias [?]30 seconds during a 90-day blanking period.

Results: A total of 697 patients (paroxysmal 51\%) were enrolled. After the PSM, both groups included 229 patients. The incidence of ERAT was significantly lower in AI group than CF group (21.5\% vs. $36.1 \%$, $\mathrm{P}<0.001)$. The rate of $\mathrm{PV}$ reconnection was lower in AI group than $\mathrm{CF}$ group $(45.8 \%$ vs. $71.4 \%, \mathrm{P}=0.028)$. Multivariate analysis demonstrated that AI-guided PVI was independently correlated with the incidence of ERAT $(\mathrm{OR}=0.415,95 \% \mathrm{CI}=0.269-0.639, \mathrm{P}<0.001)$.

Conclusion: AI-guided PVI can reduce ERAT as compared to conventional CF-guided PVI.

\section{Key words}

Atrial fibrillation; Pulmonary vein isolation; Radiofrequency catheter ablation; Ablation index; Early recurrence of atrial tachyarrhythmias

\section{Introduction}

Pulmonary vein isolation (PVI) has evolved into a widely accepted and effective strategy for the treatment of atrial fibrillation $(\mathrm{AF})$, which is recommended by the latest guidelines. ${ }^{1}$ However, we occasionally experience recurrences of atrial tachyarrhythmias after a successful PVI. Early recurrences of atrial tachyarrhythmias (ERATs) during a 90-day blanking period occur in up to $50 \%$ of patients after a PVI. ${ }^{2,3}$ The pathophysiological mechanisms of ERATs are attributed to acute inflammatory changes due to energy deliveries, delayed effects of ablation due to lesion consolidation, and reconnections of isolated PVs. ${ }^{3-5}$ ERATs reduce the patients' quality of life owing to various symptoms. In addition, ERATs predict late recurrences after PVI. ${ }^{2,3,6}$ Therefore, it is important to reduce the incidence of ERATs, even for short periods of time.

Ablation index (AI) is a novel marker of the lesion quality during radiofrequency catheter ablation (CA) and incorporates power in addition to contact force $(\mathrm{CF})$ and time in a weighted formula. ${ }^{7-9}$ An AI-guided AF ablation improves the incidence of late atrial tachyarrhythmia recurrences as compared to the conventional contact force (CF) guided ablation. ${ }^{8,10}$ However, little is known whether or not an AI-guided PVI can reduce ERATs.

We hypothesized that an AI-guided PVI, which is a novel technology of radiofrequency CA, could reduce the frequency of ERATs. Hence, this study aimed to clarify the effect of AI-guided PVI on ERATs as compared to the conventional CF-guided PVI.

\section{Methods}

\section{Study design and population}

This study was a single-center retrospective observational study. We enrolled consecutive patients who underwent a first-time radiofrequency CA of AF with the CARTO3 navigation system (Biosense Webster, Diamond Bar, CA, USA) at Osaka Rosai Hospital between September 2014 to August 2019. The exclusion criteria were (1) patients whom we could not follow up for at least 3 months and (2) patients with no available pre-procedural echocardiograms or laboratory data. 
We divided these patients into the two groups: patients who underwent an AI-guided PVI (AI group) and those who underwent a CF-guided PVI (CF group). Propensity score matching (PSM) was used to adjust for the patient age, sex, body mass index (BMI), body surface area (BSA), type of AF (paroxysmal or nonparoxysmal), history of heart failure, hypertension, diabetes and strokes, laboratory findings including the estimated glomerular filtration rate (eGFR) and B-type natriuretic peptide (BNP), and echocardiography parameters including the left ventricular ejection fraction (LVEF) and (left atrium) LA diameter. After the PSM, we compared the incidence of ERATs and late reconnection rate of isolated PVs during second session between $\mathrm{AI}$ and $\mathrm{CF}$ groups. Additionally, we performed univariate and multivariate analyses to examine the clinical factors correlated with the incidence of ERAT.

This study was approved by the local ethics committee (approved number: 2020-29). All patients gave their informed consent for the CA procedure.

\section{Catheter ablation procedure}

All antiarrhythmic drugs were discontinued for at least 5 half-lives before the procedures. The ablation procedures were performed under deep sedation with boluses of hydroxyzine, pentazocine, and propofol followed by a continuous infusion of propofol and dexmedetomidine. The patients received respiration control with adaptive servo ventilation (ASV). An esophageal temperature monitoring catheter was inserted via the nose. A duo-decapolar catheter (BeeAT; Japan Lifeline, Tokyo, Japan) was placed in the coronary sinus (CS) though the right internal jugular vein or right subclavian vein. A Brockenbrough puncture using an RF needle (Japan Lifeline) was performed under guidance of the Soundstar 3D Ultrasound Catheter (Biosense Webster). After the Brockenbrough puncture, 2 or 3 long sheaths (SL0; Abbott, Chicago, IL, USA) were inserted into the LA. An initial intravenous bolus of heparin (100IU $/ \mathrm{kg}$ body weight) was administered followed by a continuous infusion of heparin to maintain the activated clotting time at 300-350 seconds. One or two circular mapping catheters were placed in the superior and inferior pulmonary veins (PVs), and the ipsilateral PVs were circumferentially ablated guided by the CARTO3 mapping system. All PVI was performed with the CF-sensing open-irrigated-tip ablation catheter. The endpoint of the PVI was the achievement of bidirectional conduction block between the LA and PVs. After the achievement of the PVI, isoproterenol $(20-300 \mu \mathrm{g} / \mathrm{h})$ and $40 \mathrm{mg}$ adenosine triphosphate (ATP) were infused to examine any dormant $\mathrm{PV}$ reconnections and non-PV AF triggers. A non-PV trigger ablation was strongly encouraged. Cavotricuspid isthmus (CTI) block was created in almost all patients.

Ablation procedures performed between September 2014 and July 2018 were CF-guided, whereas those between August 2018 and August 2019 were AI-guided. In the CF-guided ablation, lesion creation was guided by $\mathrm{CF}$ targets of 5-40g. Each radiofrequency application was delivered for 25 seconds with a power of up to $30 \mathrm{~W}$. In AI-guided ablation, the procedure was guided by AI target values for each lesion as follows: 450 for the anterior/roof segments and 400 for the posterior/inferior segments of the LA. In both groups, the esophageal temperature was monitored continuously during the ablation procedure to avoid any thermal injury, and the radiofrequency delivery was terminated immediately when the esophageal temperature reached $>40$.

\section{Postablation follow-up}

After the procedure, the patients underwent continuous electrocardiogram (ECG) monitoring for approximately 3 days (until discharge). The patients were followed-up at 1 and 3 months after discharge and underwent a 12 lead ECG recording. We encouraged the patients to check their pulse rate and rhythm every day and to visit our hospital if they experienced any irregular pulses or chest discomfort. ERATs after the ablation were defined as any recurrence of atrial tachyarrhythmias of $>30$ seconds during the first 90 days of follow up. No antiarrhythmic drugs were administered until ERAF occurred.

\section{Statistical analysis}

EZR version 1.40 (Saitama Medical Center, Jichi Medical University, Saitama, Japan) was used for the statistical analysis, which is a graphical user interface for R (The R Foundation for Statistical Computing, 
Vienna, Austria). The continuous variables were expressed as the mean \pm standard deviation. Two-group comparisons were analyzed using a Student's t-test or Mann-Whitney U test. Categorical variables were expressed as the number (percentage), which was compared using a Fisher's exact test or chi-squared test. In the multivariate analysis, the age, sex, and clinical factors that could influence ERATs were included.

\section{Results}

\section{Patients characteristics}

During this study period, a total of 697 patients (paroxysmal 51\%) underwent a first-time radiofrequency PVI guided by CARTO3 navigation system. Sixteen patients were excluded from this study, because 15 patients could not be followed up for 3 months and 1 had no available pre-procedural echocardiogram or laboratory data. Four hundred forty-six patients underwent CF-guided ablation between September 2014 and July 2018, and 235 underwent AI-guided ablation between August 2018 and August 2019. Among these patients, 458 patients (AI group, $n=229$; CF group, $n=229$ ) were selected by PSM. A flowchart of the study population is shown in Figure 1.

After the PSM, there were no significant differences between the two groups regarding the baseline patient characteristics, type of AF, medical history, CHADS2VASc score, laboratory findings, and echocardiographic parameters (Table 1 ).

\section{Procedure characteristics}

All patients underwent a PVI. Approximately $85 \%$ of the patients underwent a CTI block line ablation in both groups. There were no significant differences with regard to superior vena cava (SVC) isolation and other non-PV foci ablation between the two groups (Table 2).

Procedure time and total ablation time were significantly shorter in AI group than CF group. Total number of energy applications was smaller in AI group than CF group. There were no significant differences regarding procedural complication.

\section{ERATs after the procedure}

After the PSM, the incidence of ERAT was significantly lower in AI group than CF group (22.3\% vs. $38.0 \%$, $\mathrm{P}<0.001$, Figure 2). The analyses of each AF subtype revealed that AI group had a significantly reduced incidence of ERAT in both the paroxysmal AF (PAF) and non-PAF patients $(15.2 \%$ vs. $31.2 \%, \mathrm{P}=0.006$; $28.2 \%$ vs. $44.2 \%, \mathrm{P}=0.011$, respectively, Figure 2 ).

\section{Findings after the second session}

Among the CF group patients with ERAT, 70 patients underwent a second session of CA for late recurrences of AF, whereas 24 patients underwent second sessions among those in AI group. The rate of a PV reconnection was lower in AI group than CF group (45.8\% vs. $71.4 \%, \mathrm{P}=0.028$, Figure 3 ). In the analyses of each PV, the percentage of right superior PV (RSPV) reconnections were significantly lower in AI group than CF group ( $20.8 \%$ vs. $48.6 \%, \mathrm{P}=0.029)$.

\section{Parameters correlated with ERAT}

The univariate analysis revealed that a female sex, non-PAF, eGFR, BNP, LA diameter, and AIguided PVI were correlated with ERAT (Table 3). The multivariate analysis identified that a female sex $(\mathrm{OR}=2.257,95 \% \mathrm{CI}=1.307-3.891, \mathrm{P}=0.003)$, non-PAF $(\mathrm{OR}=1.613,95 \% \mathrm{CI}=1.001-2.591, \mathrm{P}=0.049)$, LA diameter $(\mathrm{OR}=1.040,95 \% \mathrm{CI}=1.000-1.080, \mathrm{P}=0.043)$ and AI-guided PVI $(\mathrm{OR}=0.415,95 \% \mathrm{CI}=0.269-0.639$, $\mathrm{P}<0.001$ ) were associated with the incidence of ERAT (Table 3).

\section{Discussion}

Main findings 
The main findings of this study were as follows: (1) AI-guided PVI could reduce the frequency of ERATs more than CF-guided PVI in both the PAF and non-PAF patients; (2) the number of PV reconnections in the second CA session was significantly smaller in AI-guided PVI group than the CF-guided PVI group; and (3) AI-guided PVI was independently associated with the incidence of ERAT in addition to the sex, LA diameter and type of AF.

\section{Favorable Effects of AI on ERATs}

In this study, we revealed that AI-guided PVI could reduce ERATs more than CF-guided PVI. Tokuda et al. reported that the incidence of ERAT after PVI for PAF was similar between Cryoballoon ablation and conventional radiofrequency CA (21\% vs. $27 \%, \mathrm{p}=0.15) .{ }^{11}$ We considered that AI-guided PVI was the most effective modality for reducing ERATs in PAF patients (15.2\%).

Preclinical studies in an animal model have demonstrated that AI accurately predicts the depth of ablation lesions. ${ }^{9}$ AI-guided PVI can produce a higher impedance drop, and AI plays a role as a parameter of the effectiveness of a lesion formation. ${ }^{12}$ Several reports have demonstrated that AI-guided PVI could achieve a durable PVI and reduce late PV reconnections, ${ }^{13}$ which is consistent with our results. We considered that AI could reduce late PV reconnections, which could lead to reduction in ERATs.

In our study, AI-guided PVI decreased late PV reconnections, especially RSPV. Septopulmonary bundle runs through the RSPV ostium, which is relatively thick LA muscular bundle and renders RSPV isolation difficult. ${ }^{14}$ We suggested that AI-guided ablation could form transmural ablation lesion at this site, that lead to less reconnection of RSPV. Furthermore, the localization of focal AF triggers occurs frequently in RSPV. ${ }^{15}$ On the other hand, the rate of left PV reconnections was similar between AI group and CF group, which could be explained by the fact that we attenuated the radiofrequency delivery to the LPV posterior wall to prevent esophageal injury in both 2 groups.

\section{AI-guided PVI for non-PAF patients}

Our study revealed that AI-guided PVI could reduce ERATs in non-PAF patients as well as PAF patients. CA of non-PAF is more challenging and is correlated with worse outcomes than that for PAF. ${ }^{16} \mathrm{We}$ occasionally experience that ERATs persist for a long period of time and turn into late recurrences in non-PAF patients. Themistoclakis et al. reported that non-PAF was an independent predictor of ERAT and late recurrence after a PVI. ${ }^{17}$ We believe that AI-guided PVI reduces the frequency of ERATs, which improves the clinical outcomes after PVI.

\section{Predictors of ERATs}

AI-guided PVI, sex, LA diameter, and type of AF were independent predictors of ERAT. It has been reported that the AF duration, non-PAF, history of hypertension, LA enlargement, and lack of an SVC isolation were predictors of ERAT. ${ }^{17}$ There are few reports about the correlation between ERATs and a female sex. However, several reports have demonstrated that a female sex is a predictor of an arrhythmia recurrence after a PVI. ${ }^{18,19}$ We consider that a female sex could be a risk factor of ERAT as well as of a late recurrence.

\section{Clinical implications}

ERATs are strong predictor of late recurrences. ${ }^{2,3}$ There have been several reports that the incidence of late recurrences is significantly higher in patients with ERATs than in those without, ${ }^{5,6,20,21}$ which may be due to the recovery of the LA-PV connections or presence of incomplete ablation lesions. Our study demonstrated that AI-guided PVI could reduce ERATs and chronic PV reconnections after PVI more than could CF-guided PVI. We suggested that during PVI with radiofrequency CA, we should monitor AI during the ablation to create appropriate ablation lesions, which would lead to a durable PVI and decrease in ERATs.

On the other hand, ERAT can lead to an exacerbation of the patients' quality of life. It is our belief that we should perform AI-guided PVI for AF in patients with severe symptoms, especially in those with tachycardia induced cardiomyopathy associated with heart failure or bradycardia-tachycardia syndrome with syncope. 


\section{Conclusion}

AI-guided PVI could suppress the incident of ERATs more than conventional CF-guided PVI. The reduction in ERATs could contribute to better clinical outcomes and improve patients' quality of life.

\section{Acknowledgements}

We thank Keiji Yamamoto, Atsushi Shiono, and Tomoyoshi Morioku for their technical assistance during the electrophysiological study and John Martin for his linguistic assistance.

\section{References}

1. Kirchhof P, Benussi S, Kotecha D, et al. 2016 ESC Guidelines for the management of atrial fibrillation developed in collaboration with EACTS.Eur Heart J. 2016;37(38):2893-2962.

2. Calkins H, Hindricks G, Cappato R, et al. 2017 HRS/EHRA/ECAS/APHRS/SOLAECE expert consensus statement on catheter and surgical ablation of atrial fibrillation. Heart Rhythm.2017;14(10):e275-e444.

3. Themistoclakis S, China P. Early Recurrences of Atrial Tachyarrhythmias After Ablation of Atrial Fibrillation: How Long Do We Have to Be Blind? JACC Clin Electrophysiol. 2017;3(6):577-579.

4. O'Donnell D, Furniss SS, Dunuwille A, Bourke JP. Delayed cure despite early recurrence after pulmonary vein isolation for atrial fibrillation. Am J Cardiol. 2003;91(1):83-85.

5. Das M, Wynn GJ, Morgan M, et al. Recurrence of atrial tachyarrhythmia during the second month of the blanking period is associated with more extensive pulmonary vein reconnection at repeat electrophysiology study. Circ Arrhythm Electrophysiol. 2015;8(4):846-852.

6. Andrade JG, Khairy P, Verma A, et al. Early recurrence of atrial tachyarrhythmias following radiofrequency catheter ablation of atrial fibrillation. Pacing Clin Electrophysiol. 2012;35(1):106-116.

7. Das M, Loveday JJ, Wynn GJ, et al. Ablation index, a novel marker of ablation lesion quality: prediction of pulmonary vein reconnection at repeat electrophysiology study and regional differences in target values. Europace. 2017;19(5):775-783.

8. Hussein A, Das M, Chaturvedi V, et al. Prospective use of Ablation Index targets improves clinical outcomes following ablation for atrial fibrillation. J Cardiovasc Electrophysiol. 2017;28(9):1037-1047.

9. Nakagawa H, Ikeda A, Govari A, et al. Abstract 12104: Prospective Study Using a New Formula Incorporating Contact Force, Radiofrequency Power and Application Time (Force-Power-Time Index) for Quantifying Lesion Formation to Guide Long Continuous Atrial lesions in the Beating Canine Heart. Circulation. 2013;128(suppl_22):A12104-A12104.

10. Pranata R, Vania R, Huang I. Ablation-index guided versus conventional contact-force guided ablation in pulmonary vein isolation - Systematic review and meta-analysis. Indian Pacing Electrophysiol J. 2019;19(4):155-160.

11. Tokuda M, Yamashita S, Matsuo S, et al. Clinical significance of early recurrence of atrial fibrillation after cryoballoon vs. radiofrequency ablation-A propensity score matched analysis. PLoS One. 2019;14(7):e0219269.

12. Ullah W, Hunter RJ, Finlay MC, et al. Ablation Index and Surround Flow Catheter Irrigation: Impedance-Based Appraisal in Clinical Ablation. JACC Clin Electrophysiol. 2017;3(10):1080-1088.

13. Hussein A, Das M, Riva S, et al. Use of Ablation Index-Guided Ablation Results in High Rates of Durable Pulmonary Vein Isolation and Freedom From Arrhythmia in Persistent Atrial Fibrillation Patients: The PRAISE Study Results. Circ Arrhythm Electrophysiol.2018;11(9):e006576.

14. Ho SY, Sanchez-Quintana D, Cabrera JA, Anderson RH. Anatomy of the left atrium: implications for radiofrequency ablation of atrial fibrillation. J Cardiovasc Electrophysiol. 1999;10(11):1525-1533. 
15. Haissaguerre M, Jais P, Shah DC, et al. Spontaneous initiation of atrial fibrillation by ectopic beats originating in the pulmonary veins. N Engl J Med. 1998;339(10):659-666.

16. Verma A, Jiang CY, Betts TR, et al. Approaches to catheter ablation for persistent atrial fibrillation. $N$ Engl J Med.2015;372(19):1812-1822.

17. Themistoclakis S, Schweikert RA, Saliba WI, et al. Clinical predictors and relationship between early and late atrial tachyarrhythmias after pulmonary vein antrum isolation. Heart Rhythm. 2008;5(5):679-685.

18. Sugumar H, Nanayakkara S, Chieng D, et al. Arrhythmia recurrence is more common in females undergoing multiple catheter ablation procedures for persistent atrial fibrillation: Time to close the gender gap.Heart Rhythm. 2019.

19. Schreiber D, Rostock T, Frohlich M, et al. Five-year follow-up after catheter ablation of persistent atrial fibrillation using the stepwise approach and prognostic factors for success. Circ Arrhythm Electrophysiol. 2015;8(2):308-317.

20. Liang JJ, Elafros MA, Chik WW, et al. Early recurrence of atrial arrhythmias following pulmonary vein antral isolation: Timing and frequency of early recurrences predicts long-term ablation success. Heart Rhythm. 2015;12(12):2461-2468.

21. Willems S, Khairy P, Andrade JG, et al. Redefining the Blanking Period After Catheter Ablation for Paroxysmal Atrial Fibrillation: Insights From the ADVICE (Adenosine Following Pulmonary Vein Isolation to Target Dormant Conduction Elimination) Trial. Circ Arrhythm Electrophysiol. 2016;9(8).

\section{Figure Legends}

FIGURE 1. Flowchart of this study

$\mathrm{AI}=$ ablation index, $\mathrm{AF}=$ atrial fibrillation, $\mathrm{CF}=$ contact force

FIGURE 2. The percentage of ERATs

$\mathrm{AI}=$ ablation index, $\mathrm{CF}=$ contact force, $\mathrm{EART}=$ =arly recurrence of atrial tachyarrhythmias, $\mathrm{PAF}=$ paroxysmal atrial fibrillation

FIGURE 3. The rate of PV reconnections during the second session

$\mathrm{LSPV}=$ lest superior pulmonary vein, LIPV=left inferior pulmonary vein, RSPV=right superior pulmonary vein, $\mathrm{RIPV}=$ right inferior pulmonary vein

\section{Conflict of interest statement}

The authors declare no conflict of interests for this article.

Funding

None.

\section{Hosted file}

Tables.docx available at https://authorea.com/users/311850/articles/479808-ablation-indexguided-pulmonary-vein-isolation-can-reduce-early-recurrences-of-atrial-tachyarrhythmias 

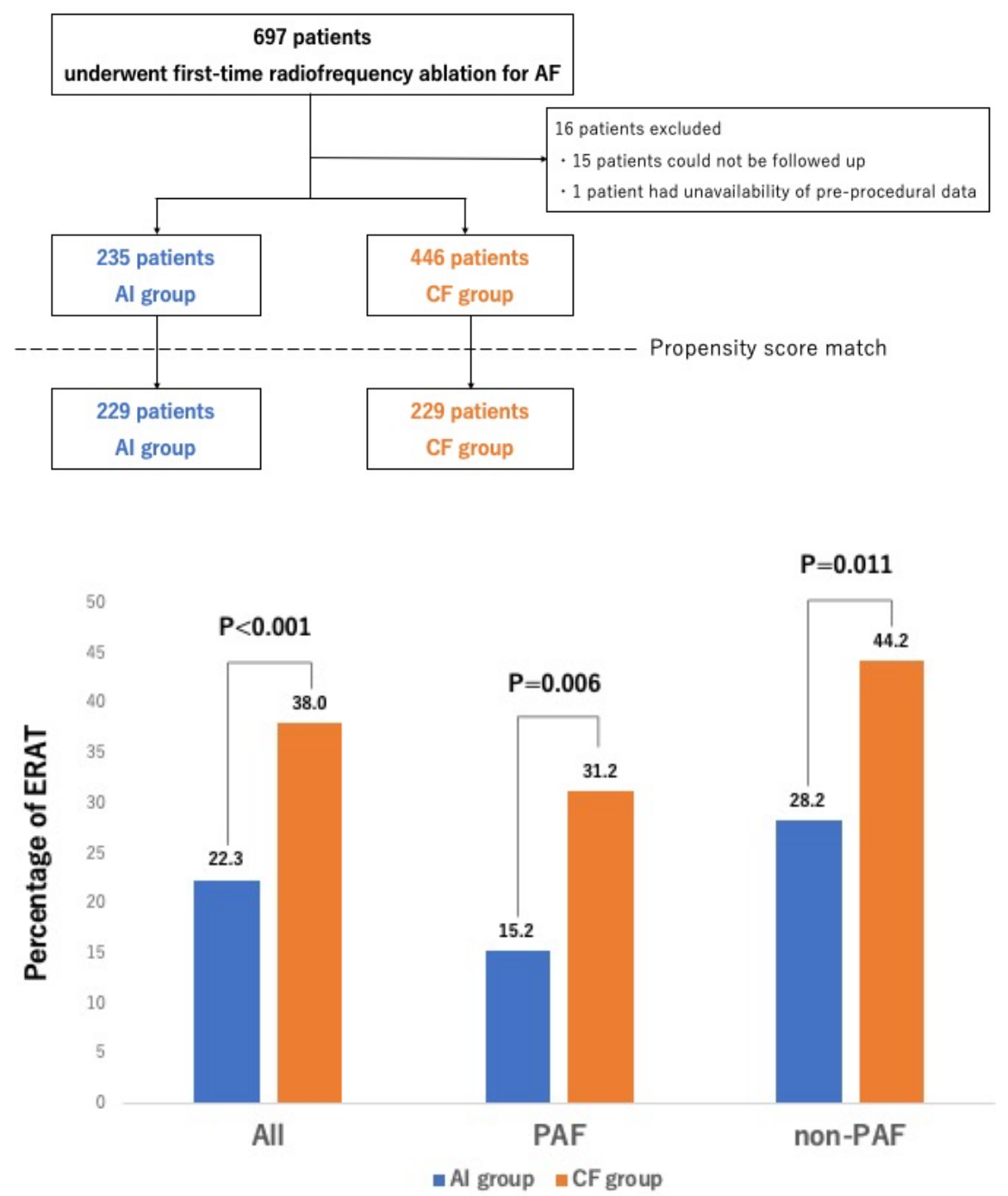


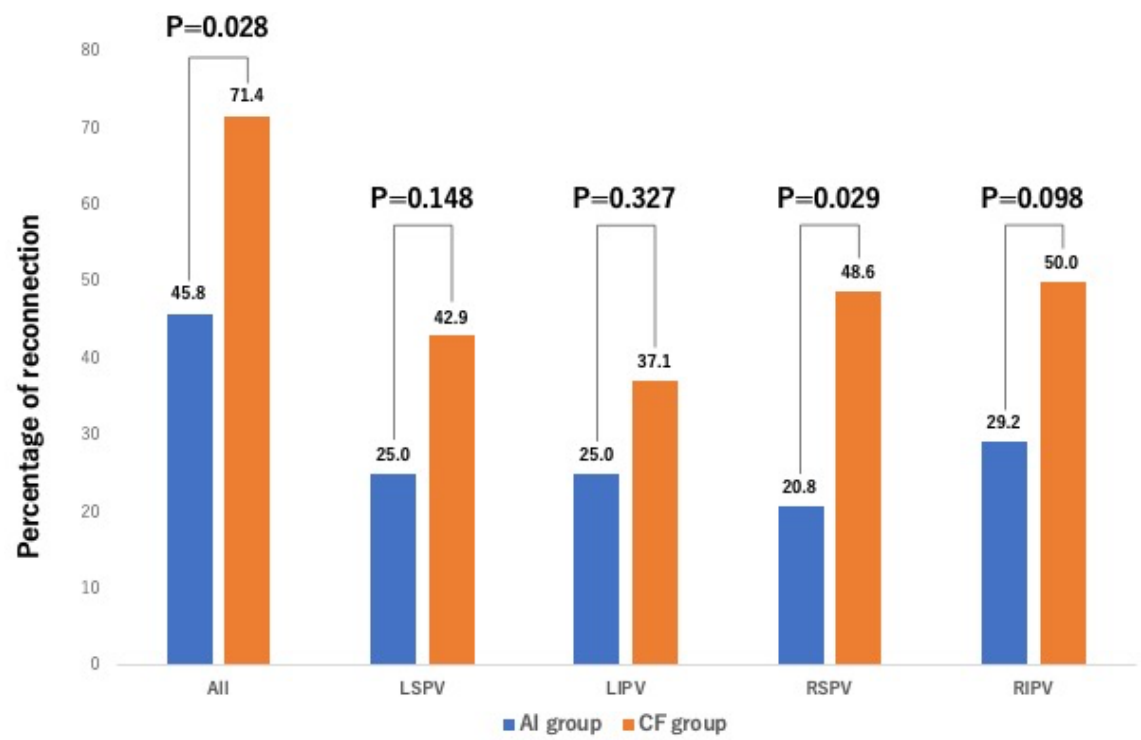

